\title{
Souvenirs des guerres napoléoniennes: points de vue des blessés et de leurs médecins
}

\author{
Par B. Rüttimann
}

Les grands noms de la médecine sont à l'histoire médicale ce que les chefs illustres sont à l'histoire générale. A l'exception des autobiographies et des Mémoires, il est assez rare que soit évoqué le sort d'un individu, d'un simple bourgeois, d'un pauvre malade ou d'un soldat blessé.

Sur le plan médical, pour nous faire une idée juste de la gravité d'une affection et de la qualité d'un traitement, nous ne nous contentons pas d'un seul rapport, soit du malade soit de son médecin. Il nous faut au moins deux témoignages différents.

La période de la Révolution française et de l'Empire est très riche en Mémoires. Un collectionneur et lecteur passionné a la chance de trouver un compte rendu médical précis rédigé par deux auteurs différents au moins, parfois même par le blessé lui-même et son médecin. Il s'agit alors de confronter ces documents, d'en étudier au mieux possible leur contexte, pour se faire ainsi une idée approfondie et équilibrée de l'évènement. Par ce procédé nous sommes à même de nous représenter avec quelque certitude le sort de patients d'il y a presque 200 ans.

\section{Daumesnil: «Ange gardien» et «Jambe de bois»}

Le major Yrieix Daumesnil (1776-1832) des chasseurs de la garde impériale était connu sous le nom «d'ange gardien de Napoléon» parce que, «simple guide du général Bonaparte, à Saint-Jean-d'Acre, il avait exposé ses jours pour sauver ceux de son général» en couvrant «de son corps celui qui plus tard devait être son empereur» (Parquin Mém., Baschet 1938). A la bataille de Wagram (1809) il reçut, comme le constatait le chirurgien en chef de la garde Dominique Jean Larrey (1766-1842), «un boulet de canon qui lui brisa les deux os de la jambe droite, à son articulation tibio-tarsienne. Cette blessure commandait l'amputation du membre: je la pratiquai immédiatement sur le champs de bataille» (Larrey Mém. III et Rel.). Le célèbre chirurgien militaire avait adopté et perfectionné le procédé opératoire de Petit (Larrey Mém. II et III, Larrey Clinique, Richerand 1825, SabatierDupuytren 1832) qui lui garantissait d'excellents résultats. 
Il en fut de même pour Daumesnil, dont le moignon se cicatrisa promptement. Nous en avons une preuve dans l'épisode suivant: Le frère du docteur Poumiès de la Siboutie «alla le voir après le malheureux accident... lui fit ses compliments de condoléances et lui dit toute la peine qu'il en avait éprouvée: - Ma foi! dit Daumesnil, ce n'est pas un malheur aussi grand qu'on se l'imagine. Je portais à cette jambe depuis plus de dix ans une plaie qui me faisait horriblement souffrir. Me voilà guéri et je ne sens plus rien!» (Poumiès Mém.). Dans la collection d'autographes de Larrey se trouvait la lettre suivante: «Tout va bien, mon cher Larrey. Mon bobo sera bientôt guéri, et il ne me restera plus de souvenir de mon accident qu'une jambe en chair et en os de moins et une jambe de bois de plus. Je ne m'en suis pas mal tiré. Reçois mes remerciements par écrit. Je ne sais quand je pourrai te les adresser de vive voix. Daumesnil. Vienne, 30 août 1809» (Poumiès Mém.).

On peut se représenter cette prothèse de bois un peu comme la «Iambe de bois pour les pauures» qu'avait déjà dessiné Ambroise Paré (1585) et qui restait le modèle usuel jusqu'à la fin du dernier siècle (fig. 1). Daumesnil était alors surnommé aussi «la Jambe de bois» (Larrey Rel.). Lors de grandes et solennelles occasions pourtant, devenu général de division et Baron d'Empire, il portait une prothèse très élégante de construction métallique, munie d'une articulation de genou (Huard 1970). Pour récompenser ses éminents services, Napoléon «donna au général Daumesnil le commandement de Vincennes, où il se couvrit de gloire plus tard, lors de nos revers, par la réponse énergique qu'il fit à l'ennemi, qui, maître de Paris, le sommait de se rendre: - Dites aux alliés que lorsqu'ils me rendront ma jambe, je leur donnerai les clefs de la place; jusque-là, qu'ils passent au large...» (Parquin Mém.). Le lac situé à proximité du château de Vincennes porte le nom de Daumesnil en souvenir de la défense hérö̈que de cette place.

\section{L'épaule broyée du général Thiébault}

Longuement et avec une certaine complaisance, Dieudonné-Paul-Charles Thiébault (1769-1846) nous informe, dans les moindres détails, de la blessure dont il fut victime à la fin de la mémorable journée d'Austerlitz (2 décembre 1805, fig. 2). Il nous montre par là aussi l'ampleur des soins prodigués aux «officiers de l'Empereur» malades ou blessés. La correspondance de Larrey dévoile même des abus (Triaire 1902); aussi Thiébault s'était-il attaché à son propre service, et à lui seul, un chirurgien d'armée: il 


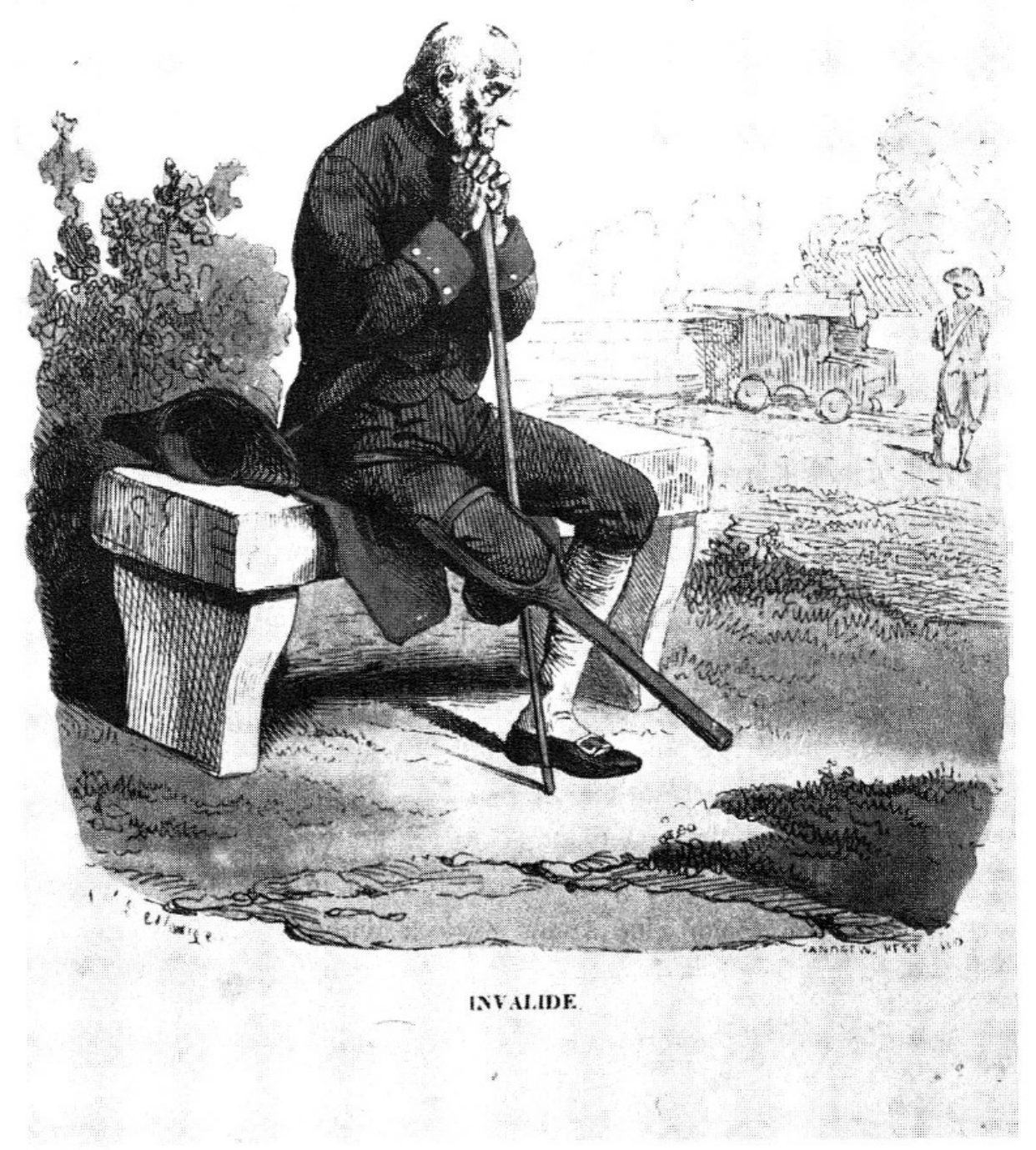

Fig. 1. Invalide avec une prothèse de bois «à marcher sur le genou» (Collection de l'Institut d'Histoire de la Médecine, Université de Zurich)

allait ainsi à l'encontre des directives de Larrey, ce qui ne manqua pas d'influencer la rédaction de leurs souvenirs. Le récit de ce personnage cultivé 
et prosaïque nous est d'une grande utilité: il nous procure un rapport circonstancié vu sous l'angle du patient. Dans les Mémoires de cette période on ne rencontre pas de récits aussi détaillés.

Thiébault montait avec un peloton d'hommes à l'assaut d'une batterie russe. «...je me retournai à moitié pour leur crier d'accélérer la course... et je fus blessé par une balle de mitraille qui, après m'avoir broyé l'épaule droite, me brisa le sternum en deux endroits et traversa la clavicule» (Thiébault Mém. III). Présentant le flanc à l'ennemi, le projectile était entré dans l'articulation scapulo-humérale et avait poursuivi sa course à travers les

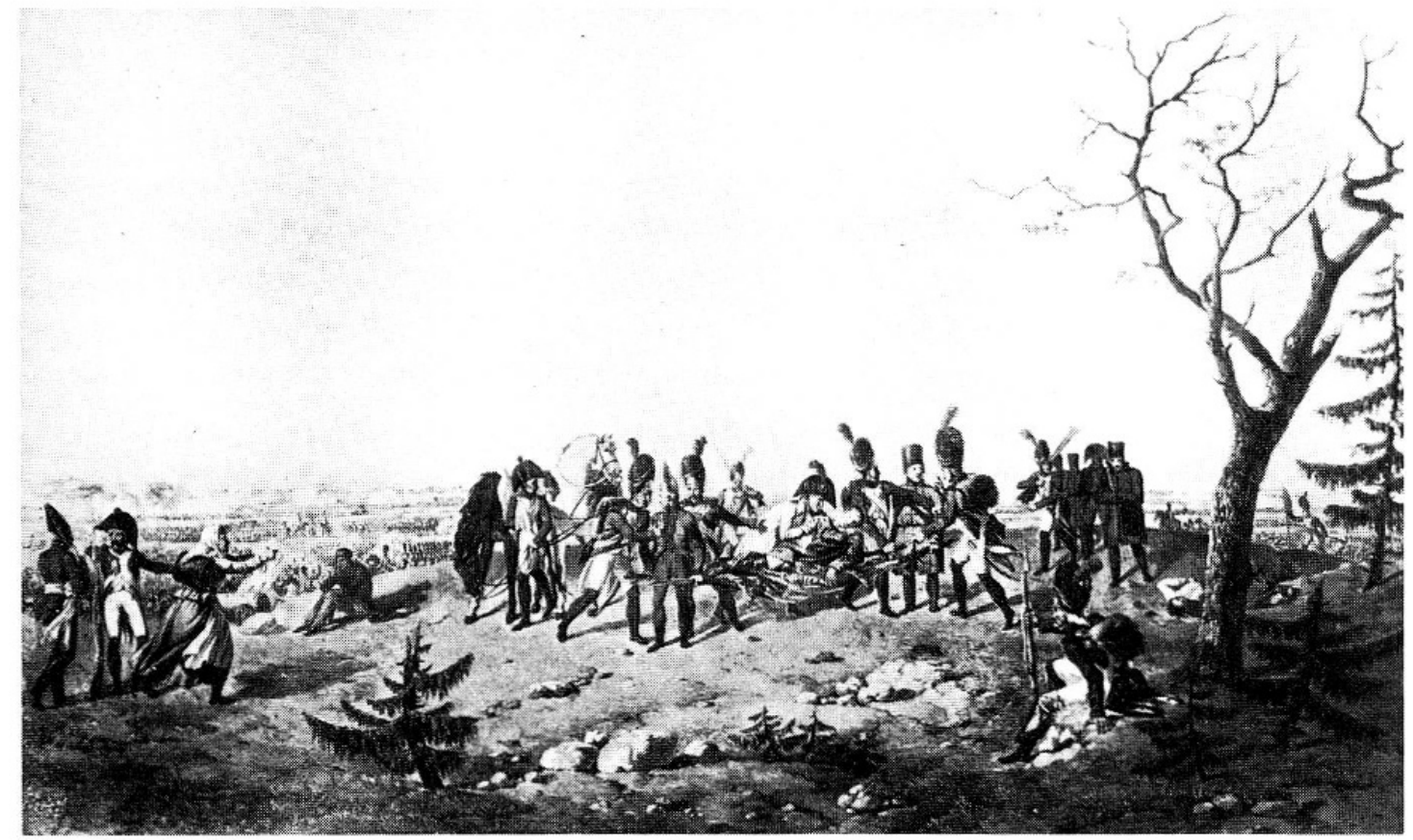

Fig. 2. Le général Thiébault blessé à la bataille d'Austerlitz (Thiébault Mém. III)

tissus moux de l'épaule pour finalement sortir du thorax en fracassant la clavicule et le sternum. La gravité des lésions ne fait aucun doute, et l'auteur des Mémoires n'exagère en rien la description de sa blessure: Triaire (1902) lui reproche pourtant une parfaite exagération décrivant un trajet inverse de la balle, responsable, selon lui, de lésions moins graves. Triaire s'était en effet appuyé sur une notice de Larrey qui écrivait 35 ans plus tard, en 1841: «Une balle lui avait traversé l'épaule gauche [sic], du centre de la clavicule, qui fut fracturée, au sommet du scapulum»(Larrey Rel.). Erreur pardonnable quant au côté touché mais inexcusable pour l'appréciation du traumatisme. Larrey lui-même avait débridé et sondé les plaies d'entrée et de sortie pour 


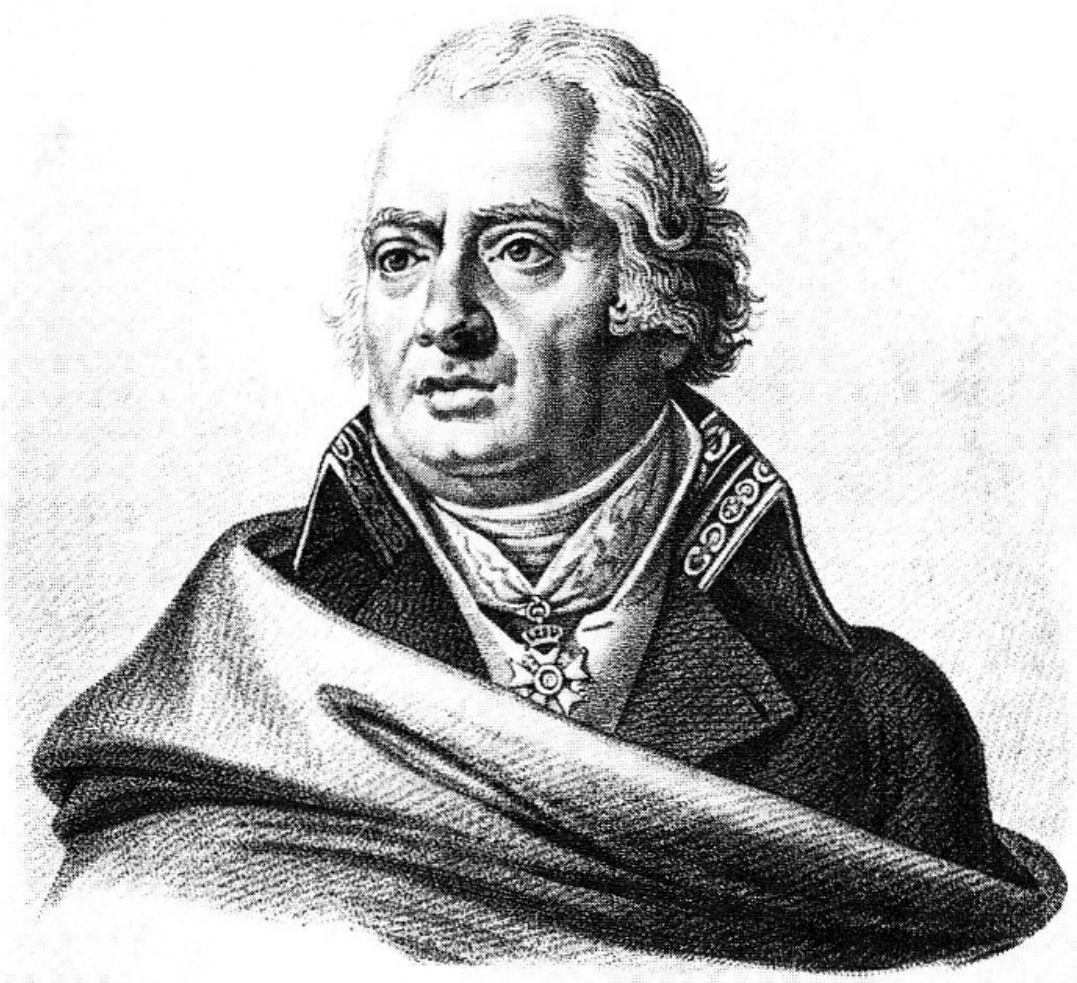

Fig. 3. Pierre-François Percy, chirurgien en chef de la Grande Armée (Collection de l'Institut d'Histoire de la Médecine, Université de Zurich)

démontrer au patient qu'il n'y avait eu qu'un seul projectile. Voici le passage correspondant dans les Mémoires de Thiébault: «On m'avait laissé dans cette erreur; mais M.Larrey, qui s'est toujours mieux entendu à couper des membres qu'à ménager un malade, se trouvant un matin seul à mon pansement, me désabusa au très grand mécontentement de M. Percy, et cela en me fourrant dans l'épaule une sonde que je fus désagréablement surpris de me voir sortir sous le menton.» Or la règle admise depuis l'antiquité et énoncée par Ambroise Paré (1545) veut que le sondage se fasse selon la trajectoire du projectile. La reminiscence du patient concorde donc avec cette loi et met en défaut la mémoire du chirurgien qui ne manquait certainement pas aux exigences de son art.

Décidément Thiébault n'aimait pas Larrey; il vénérait par contre PierreFrançois Percy (1754-1825), chirurgien en chef de la Grande Armée, plus âgé, cultivé et plus affable (fig. 3). «A dater de ce jour, M. Percy me témoigna 
l'intérêt le plus tendre, et, ne se bornant plus à ses visites du matin, il venait tous les soirs passer une heure ou une heure et demie avec moi. Ce qui ces causeries me firent de bien est indicible; elles me charmaient et me ravivaient» (Thiébault Mém. III). Aux yeux d'un malade exigeant, il ne suffit donc pas d'être un chirurgien habile et illustre si l'on est toujours pressé!

Le «Trente-sixième Bulletin de la Grande Armée, Schonbrünn le 23 frimaire an 14» confirmait que le général Thiébaut (sic) avait été dangereusement blessé, mais se rétablissait (Campagnes 1805). La grande crainte de Thiébault ne s'était pourtant dissipéé que depuis peu, «car d'après l'opinion générale, indépendamment de ce que sept fractures ont toujours de grave, et particulièrement en ce qui touche à la poitrine, il parut impossible que, dans le trajet de neuf pouces franchi par le projectile depuis son entrée dans l'épaule droite jusqu'à sa sortie par le sternum, il n'y ait pas eu quelque artère de coupée...» (Thiébault Mém. III).

Relevons une observation valable encore de nos jours lors d'un traumatisme de ce genre: «Quand le bras est cassé, on l'attache au corps, quand la clavicule est cassée, le bras devient un point d'appui; mais quand le sternum, la clavicule et l'épaule sont brisés, on ne sait plus sur quoi s'étayer pour empêcher la vacillation des os fracturés, et cette situation diabolique était la mienne. - Il fallait que je découvrisse un moyen de bandage, puisque les premiers chirurgiens du monde ne le trouvaient pas; j'imaginai donc une gaine en cuir de buffle, taillé, percée et ajustée de manière à me contenir le bras entier dans la position voulue, et le tout fut fixé autour du corps par dix [des?] bandes de toile; il n'y eut plus une oscillation à craindre» (Thiébault Mém. III). Que n'a-t-il laissé un dessin de son orthèse: on en ferait toujours bon usage!

\section{Le Général Rapp: vingt-trois blessures}

«Le général comte Rapp, ancien aide-de-camp de mon illustre ami le Général Desaix, a été pansé par moi de plusieurs blessures légères qu'il avait reçues dans divers combats, et notamment à la bataille d'Austerlitz» (Larrey Rel.).

La blessure qui frappa Jean Rapp (1772-1821) à Austerlitz n'était sûrement pas la plus grave mais sans aucun doute la plus spectaculaire: «Les canons, le prince Repnin, étaient dans nos mains; malheureusement nous avions un bon nombre d'hommes hors de combat, le colonel Morland n'était plus, et j'avais moi-même un coup de pointe dans la tête. J'allai rendre 
compte de cette affaire à l'empereur; mon sabre à moitié cassé, ma blessure, la sang dont j'étais couvert, un avantage décisif remporté avec aussi peu de monde sur l'élite des troupes ennemies, lui inspirèrent l'idée du tableau qui fut exécuté par Gérard» (Rapp Mém.). Ce tableau est magnifique mais ne se prête malheureusement pas à une simple reproduction. Il brille de la joie et de l'exaltation du guerrier intrépide qu'était Rapp. (La peinture du baron Gérard se trouve au Musée de Versailles.)

Cet officier ne se souciait guère d'une plaie ou d'une cicatrice de plus, tant qu'il entrevoyait de l'avancement, de nouveaux faits d'armes et la gratitude de l'Empereur. L'œuvre de Gérard cadre dans ce sens tout à fait avec l'Epopée entière de même qu'avec la Légende napoléonienne. - Rapp à la fin de 1806 décrit une partie de ses blessures de guerre couvrant les campagnes de la Révolution et de l'Empire: «J'avais été blessé quatre fois dans nos premières campagnes aux armées du Rhin, sous Custine, Pichegru, Moreau, Desaix; deux fois sous les ruines de Memphis, et dans la Haute-Egypte sous les murs de Thèbes; à la bataille d'Austerlitz et à Golymin: je le fus encore quatre fois, comme on le verra par la suite, à la Moskwa. - De Golymin je fus transporté à Varsovie. Napoléon y entra le $1^{\mathrm{er}}$ janvier [1807]; il me fit l'honneur de venir me voir. «Eh bien, Rapp, tu es encore blessé, et toujours au mauvais bras.) C'était la neuvième blessure que j'avais reçue à ce bras seulement, qu'il appelait le bras malheureux. «Cela n'est pas étonnant, sire; toujours des batailles!〉 (Nous finirons, répliqua-t-il, quand nous aurons quatre-vingts ans.»» (Rapp Mém.).

Ni Rapp ni son Empereur n'atteignirent cet âge: Rapp décèdera d'un cancer du pylore à 49 ans seulement (Six 1934), la même année que Napoléon à l'île de Sainte-Hélène.

\section{Le comte de Ségur: une balle dans le dos}

Au comte Philippe-Paul de Ségur (1780-1873, fig.4) nous devons avant tout son «Histoire de Napoléon et de la Grande Armée en 1812», ouvrage d'une éminente valeur pour l'étude de la campagne de Russie. Du point de vue médical ces deux volumes, publiés pour la première fois en 1824, peuvent être complétés par les Mémoires de Larrey [1817], de Heinrich von Roos [1832] et d'Antoine Kaempfen (1962). Les Mémoires proprement dits de Ségur sont moins connus; on y trouve également les évènements de l'année 1812. Retraçant la guerre d'Espagne et plus précisément la bataille de Somosierra 
(1807), l'auteur décrit, en fin observateur et patient modèle, ses trois blessures consécutives et les soins apportés par les chirurgiens militaires.

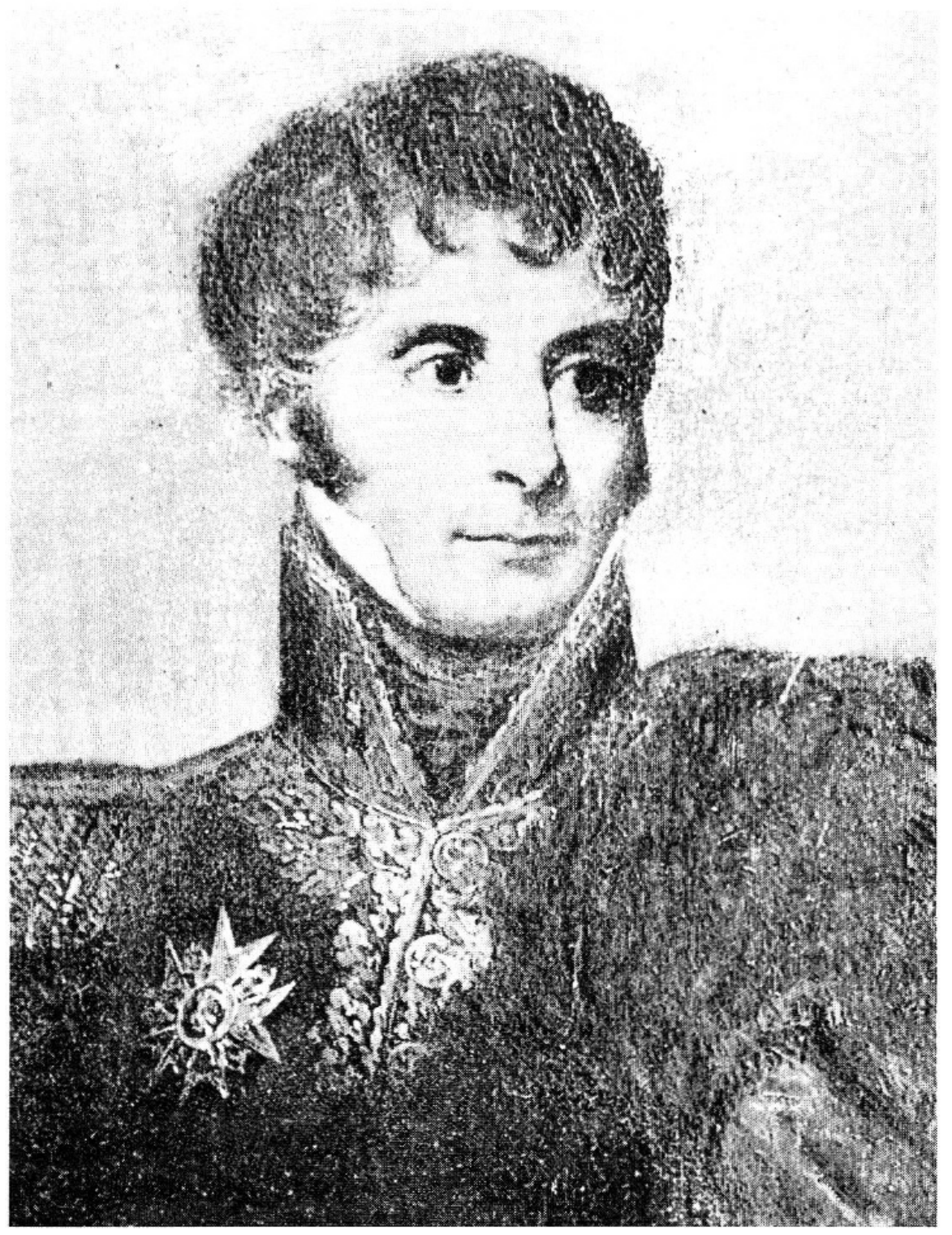

Fig. 4. Le comte Philippe-Paul de Ségur (1780-1873) par Gérard (Ségur Mém. I)

«Un biscaïen vint alors m'effleurer le cœur qu'il mit presque à découvert. Je me consultai; mais, comprenant vite qu'une telle blessure devait être mortelle ou insignifiante, et ne me sentant pas défaillir, je continuai. (Je fus cependant six mois à en guérir.) Enfin, presque au même instant, un coup de 
feu dans le côté droit m'ayant coupé la respiration, je m'arrêtai et regardai autour et derrière moi. - L'escadron presque tout entier était abattu. Enfin, parvenu au milieu des nôtres, et le péril ne me soutenant plus, je tombai dans les bras des grenadiers du $96^{\mathrm{e}}$. - Un peu plus loin, le groupe que nous formions, en passant près de l'Empereur, attira ses yeux; il s'informa. 〈Ah, pauvre Ségur! s'écria-t-il; Iwan [sic], allez vite, et sauvez-le-moi?) Je tiens ce détail d'Ywan lui-même. Ywan accourut; et, se réunissant aux grenadiers, il les aidait à me porter, lorsqu'une autre balle espagnole, venue des crêtes du défilé, me choisissant seul au milieu de toutes ces têtes penchées sur moi et qui me couvraient, les effleura sans les blesser et me traversa la cuisse droite!»

«Ywan, en me dépouillant de mes habits coupés et percés de toutes parts, comme dans une exécution militaire, quelque accoutumé qu'il fût à ces sortes d'aventures, ne pouvait retenir l'expression de son étonnement. Les contusions, la large blessure que j'avais sur le cœur, celle de la cuisse qu'il lui fallut ouvrir pour en arracher la balle, l'arrêtèrent peu. Mais à la contraction de sa figure, quand il vit le coup qui avait pénétré dans mes entrailles au-dessus [ou plutôt au-dessous] du foie, et dont il sondait vainement la profondeur, je compris qu'il perdait tout espoir de me sauver» (Ségur Mém. I). Alexandre Urbain Yvan (1765-1839) était le second chirurgien particulier de l'Empereur. Il fut bientôt aidé par Larrey qui ne voulait pas non plus répondre de la vie du patient (Ségur Mém. I). Larrey localisa l'entrée de le balle «au côté droit du bas-ventre... Le projectile pénétra profondément dans les chairs vers la région lombaire, et s'arrêta, sans doute, dans l'intervalle des apophyses transverses de l'une des vertèbres de cette région, où il est resté» (Larrey Rel.). On pansa le moribond mais on n'osa pas entreprendre l'extraction du projectil.

«C'est ici le moment de dire que M. de Ségur, laissé à Buitrago avec un coup de feu dans le bas-ventre, est dans l'état le plus fâcheux; M. Ribes est resté près de lui» (Percy Mém.). Ségur parle lui-même du séjour à Buitrago, de la surveillance et du traitement médical, non sans relever un aspect susceptible d'intéresser tout étudiant et tout médecin en début de formation: la solitude en face du danger sera d'une grande responsabilité lorsqu'il n'y a subitement plus de collègue plus âgé à appeler ou à consulter. «...j j’étais resté à Buytrago [sic] tête à tête avec mon chirurgien, c'est-à-dire à peu près avec moi seul; non pas que ce docteur fût sans mérite, l'avenir a prouvé tout le contraire; mais, trop jeune alors, et faute d'habitude ou de foi dans son art et en lui-même, il était de ceux qui craignent, en l'attaquant, d'attirer l'ennemi. 
Il n'osait rien. De peur de tuer, il laissait mourir! Il temporisait indécis, quand le blessé était aux prises avec son mal et qu'il n'y avait pas de temps à perdre! En cette occasion le timide docteur était d'autant plus encouragé dans ce système, que les derniers adieux de mes amis, et les pronostics de ses maîtres, venaient de la convaincre que j'étais sans ressource aucune» (Ségur Mém. I).

Heureusement pour lui-même, pour Napoléon et pour la postérité, de Ségur s'est bien tiré d'affaire, ne serait-ce que pour la renommée d'écrivain qu'il s'est faite à la suite de ces campagnes. L'Empereur avait régulièrement pris de ses nouvelles et s'intéressait aux progrès de sa guérison; aussi demanda-t-il un jour à Percy: «Avez-vous vu M. de Ségur? - Je n’ai pu le voir à Buitrago; mais j'appris qu'il allait bien, qu'il n'avait aucun des accidents qui annoncent la lésion des viscères et que la balle qui avait frappé la poitrine n'y avait pas pénétré. - Celle du bas-ventre y est restée. Cela ne peut-il pas entraîner des suites? - Sire, la nature se délivrera du corps étranger; le mouvement continuel des intestins chassera la balle de côté; elle se logera dans les graisses et son poids l'entraînera vers l'anus, où elle suscitera un abcès dont l'ouverture lui donnera issue» (Percy Mém.).

\section{La cause de la mort du maréchal Lannes: une amputation inutile?}

Jean Lannes (1769-1809) avait le même âge que Napoléon et il était son ami intime. Ses grandes qualités de soldat et de tacticien lui avaient valu le bâton de maréchal et le duché de Montebello. A la fin de la bataille d'Essling (1809) il venait de s'asseoir sur le bord d'un fossé, les jambes croisées l'une sur l'autre, «lorsqu'un petit boulet de trois, lancé par le canon d'Enzersdorf, arrive en ricochant et va frapper le maréchal au point où ses deux jambes se croisaient!... Nous transportâmes le maréchal à la tête de pont, où les chirurgiens en chef procédèrent à son pansement. Ces messieurs tinrent au préalable un conciliabule secret dans lequel ils furent en dissidence sur ce qu'il fallait faire. Le docteur Larrey demandait l'amputation de la jambe dont la rotule était brisée; un autre...voulait qu'on les coupât toutes les deux; enfin, le docteur Yvan, de qui je tiens ces détails, s'opposait à ce qu'il fût fait aucune amputation... Larrey était le chef du service de santé des armées; son avis l'emporta donc: une des jambes du maréchal fut amputée» (Marbot Mém. II). Marbot ne laissa pas de doute qu'il partageait l'opinion d'Yvan.

Le récit de Larrey diffère grandement des souvenirs du capitaine, plus 
tard général baron de Marbot (1782-1854), dont les Mémoires très populaires se lisent comme un roman. On en a même dressé un parallèle avec l'œuvre de Dumas. Ces Mémoires doivent donc être interprétés avec quelques réserves (Tulard 1971). Alors officier d'ordonnance, Marbot assista à la blessure de Lannes, à son évacuation, à son traitement et jusqu'à sa fin «entre ses bras» (Marbot Mém. II). Larrey a retenu de cet évènement entre autres ceci: «... je réclamai l'assistance de plusieurs chirurgiens-majors expérimentés. Nous

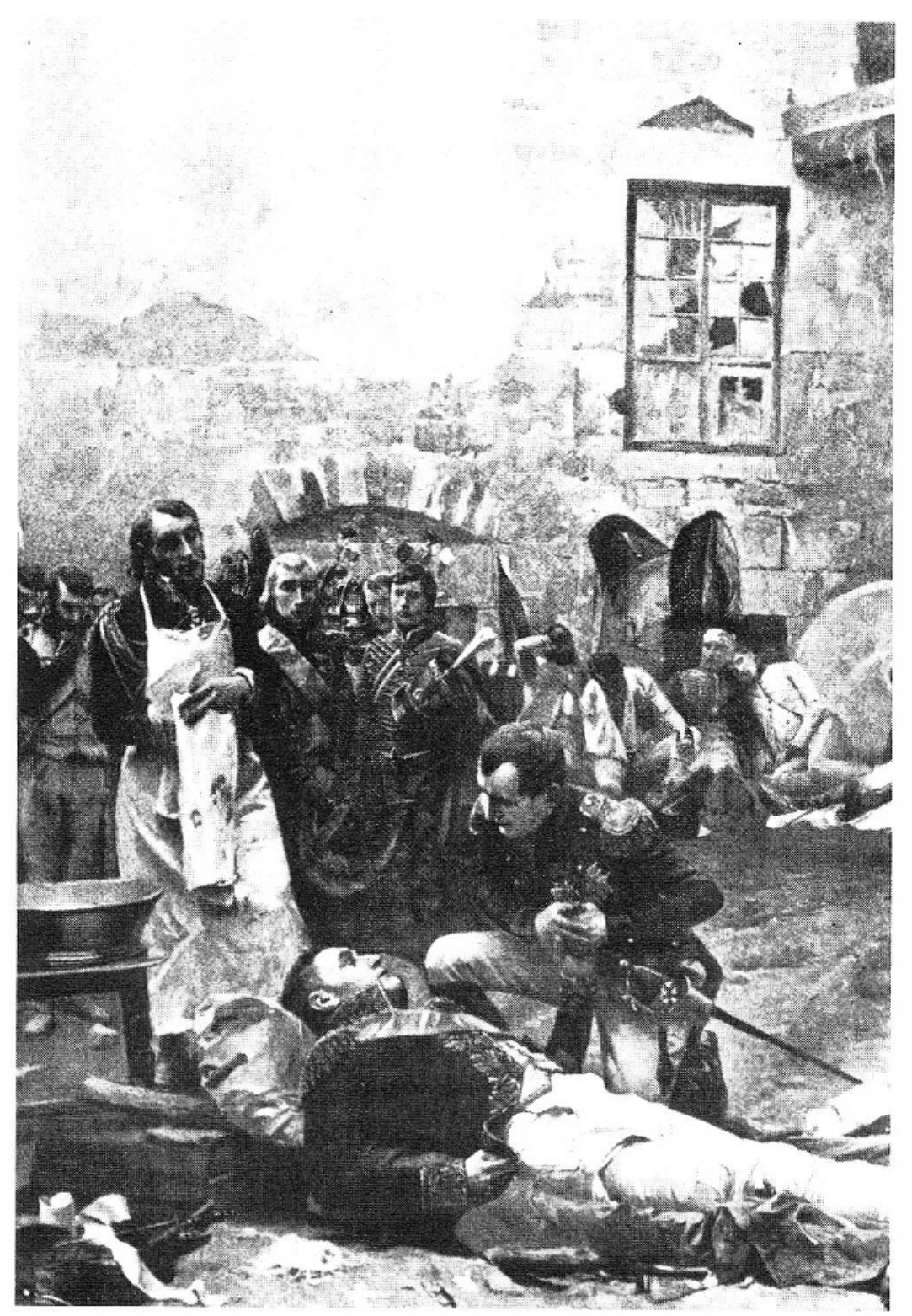

Fig. 5. Napoléon auprès du maréchal Lannes amputé par Larrey (à gauche)

(Huard op. cit. fig. 149) 
examinâmes d'abord avec le plus grand soin les deux blessures. Celle de la cuisse droite fut pansée la première avec un appareil fort simple, parce qu'elle ne nous offrit aucun accident grave. Celle du genou gauche était effrayante par le fracas des os, la déchirure des ligamens [sic], la rupture des tendons et de l'artère poplitée. Tous mes camarades reconnaissaient la nécessité de faire sur-le-champ l'amputation... et soutenu par le désir formel que manifestait le malade de subir l'opération, je me déterminai à la faire: elle fut pratiquée en moins de deux minutes...» (Larrey Mém. III, fig. 5).

L'amputation d'un membre a toujours été et restera toujours une intervention «héroique» et mutilante. Au $18^{\mathrm{e}}$ siècle sa valeur était très contestée et vivement disputée dans les écrits médicaux et devant l'Académie royale de Chirurgie. Les exigences et les expériences des guerres de la Révolution et de l'Empire mirent transitoirement fin aux débats sur l'indication et le moment propice à l'ablation d'une extremité traumatisée. Rappelons-nous qu'il n'existait aucune anesthésie proprement dite ni antisepsie ou asepsie! La distinction entre blessures de guerre et «civiles» n'avait pas encore l'importance qu'elle a gagné depuis. Pour éviter les infections incontrôlables et la «pourriture d'hôpital», les chirurgiens recouraient de plus en plus souvent au principe du «débridement préventif» pour «rendre la plaie simple», principe formulé pour la première fois en 1741 par Henri-François le Dran. Or, l'ultime conséquence du débridement est justement l'amputation. Si elle était inévitable, les chirurgiens la pratiquaient le plus tôt et le plus vite possible, souvent sur le champs de bataille même, ou dans l'ambulance établie à proximité des troupes engagées. C'est pour ses innovations dans le service de santé, le triage des blessés et leur traitement immédiat que Larrey avait mérité d'être appelé par l'Empereur «la Providence du soldat» (Bégin 1849, Brice et Bottet 1907, Rochard 1875, Rüttimann 1979 et 1985).

Il est bien possible qu'il y ait eu «dissidence» dans «le conciliabule secret» que tinrent les officiers de santé à propos des soins convenants au maréchal blessé. Mais l'insinuation de Marbot, estimant que Larrey s'imposait seulement par son rang, me semble erronée. La tenait-il peut-être d'un Yvan vexé? Toujours est-il qu'en cette année 1809 Larrey dépassait de loin tous ses collègues en expérience personnelle de chirurgie militaire. Son ami Lannes ne manquait certainement pas de connaître la réputation acquise par Larrey qui l'avait soigné bien des fois dans les campagnes précédantes. Ainsi la décision grave qui a été prise peut s'expliquer. Elle s'avère d'ailleurs juste en ce qui concerne la blessure, «car la plaie du moignon était en voie de 
guérison, lorsque la fièvre typhoïde le saisit et le fit périr le treizième jour de l'accident» (Larrey Rel.). Le typhus régnait alors dans toute l'armée et il est bien probable que le maréchal, déjà très affaibli par ses plaies, lui ait succombé. De Vienne accourut même le célèbre docteur Frank, appelé en consultation. «...il donna son approbation au traitement mis en usage, et voulut rester avec nous auprès du malade dont les forces déclinaient progressivement. Enfin, le maréchal entra dans un délire complet qui fut de courte durée, et il mourut peu d'heures après dans un état assez calme» (Larrey Mém. III).

\section{Conclusions}

Nous avons vu que «L'ange gardien de Napoléon» ne se montrait pas outre mesure affecté par la perte d'une jambe, que le général de brigade Thiébault faisait grand cas d'une blessure à l'épaule qui était néanmoins très sérieuse, que le grognard Rapp survécut à 23 blessures pour mourir prématurément d'un cancer, que le comte de Ségur atteint très gravement avait de la compassion et de la compréhension pour les angoisses et l'inexpérience d'un jeune médecin; finalement nous relevons qu'un des plus illustres maréchaux de l'Empire pouvait succomber au typhus tout aussi bien qu'à un traumatisme nécessitant une amputation.

Nous avons ainsi constaté que ces témoignages confrontés ne concordent pas toujours. La différence des points de vue du patient et du médecin l'explique, comme de nos jours. Nous aussi, nous n'avons pas encore tout à fait surmonté cette différence malgré les deux siècles qui nous séparent des expériences médicales d'un Larrey ou d'un Percy. Mais aujourd'hui comme hier, les médecins peuvent constater que leur savoir et leur bonne volonté sont généralement remarqués et appréciés par les malades. Nous l'avons démontré à propos de Larrey qui porte dans l'histoire de l'Empire et dans l'histoire de la médecine le titre de «Providence du soldat». 


\section{Mémoires cités}

Kaempfen, Antoine. Deux cahiers des «Souvenirs» du D ${ }^{\mathrm{r}}$ Antoine Kaempfen (1784-1856) publiés par Georges Foëx. Valesia, 1962, 1-120

Larrey, Dominique Jean. Mémoires de chirurgie militaire et campagnes. Tome I-III, J. Smith et F. Buisson, Paris, 1812. Tome IV, J.Smith, Paris, 1817

Larrey, Dominique Jean. Relation médicale de campagnes et voyages de 1815 à 1840.1 vol. J.B. Baillière, Paris, 1841

Marbot, Jean-Antoine-Marcellin. Mémoires du général baron de Marbot. Tome I-III, PlonNourrit, Paris 1891

Parquin, Denis-Charles. Souvenirs et campagnes d'un vieux soldat de l'Empire. 1 vol. BergerLevrault, Paris, 1892

Percy, Pierre-François. Journal des campagnes du baron Percy publié... par Emile Longin. 1 vol. Plon-Nourrit, Paris, 1904

Poumiès de la Siboutie, Louis-François. Souvenirs d'un médecin de Paris publiés par Mmes Branche et Dagoury. 1 vol. Plon-Nourrit, Paris, 1910

Rapp, Jean. Mémoires écrits par lui-même et publiés par sa famille. 1 vol. Bossange, Paris, 1823

v. Roos, Heinrich. Mit Napoleon in Russland. 1 vol. Verlag von Robert Lutz, Stuttgart, o. J. [1832 Saint-Pétersbourg].

de Ségur, Philippe-Paul. Un aide de camp de Napoléon (1800-1815). Mémoires. Tome I-III, Firmin-Didot, Paris, 1894-1895

Thiébault, Dieudonné-Paul-Charles-Henri. Mémoires du général baron Thiébault publiés... par F. Calmettes. Tome I-V, Plon, Nourrit, Paris, 1893-1895

\section{Autres sources}

Baschet R. Le général Daumesnil. Hachette, Paris, 1938

Bégin L.-J. Etudes sur le Service de santé militaire en France, son passé, son présent, son avenir. Chapitre II, de 1792 à 1834. J.-B. Baillière, Paris, 1849

Brice et Bottet. Le corps de santé militaire en France. Son évolution - ses campagnes 1708-1882. Berger-Levrault, Paris et Nancy, 1907

Campagnes de la Grande Armée et le l'Armée d'Italie, en l'an XIV (1805), ou Recueil des Bulletins etc. A la Librairie Economique, Paris, 1806

Huard P. Sciences - Médecine - Pharmacie de la Révolution à l'Empire (1789-1815). Dacosta, Paris, 1970

Larrey D.J. (Clinique chirurgicale) Chirurgische Klinik. Trad. par le $\mathrm{D}^{\mathrm{r}}$ Albert Sachs. C.Fr. Amelang, Berlin, 1831

Paré A. La Methode de traicter les playes faictes par hacquebutes et aultres bastons à feu etc. Gaulterot, Paris, 1545

Paré A. CEuvres d'Ambroise Paré. Quatrième édition. Gabriel Buon, Paris, 1585

Richerand A.-B. Histoire des progrès récens [sic] de la chirurgie. Béchet jeune, Paris, 1825

Rochard J. Histoire de la chirurgie française au XIX ${ }^{\mathrm{e}}$ siècle. J.-B. Baillière, Paris, 1875

Rüttimann B. Larreys Amputationstechnik. Gesnerus 1979, 36, 140-155 
Rüttimann B. La Providence du soldat. Schweiz. Rundschau Med. (PRAXIS) 1985, 74, $197-201$

Sabatier-Dupuytren. De la Médecine opéatoire. Nouvelle édition. Béchet jeune, Paris, 1832

Six G. Dictionnaire biographique des généraux et amiraux français (1792-1814). Saffroy, Paris, 1934

Triaire P. Dominique Larrey et les campagnes de la Révolution et de l'Empire. Mame, Tours, 1902

Tulard J. Bibliographie critique des Mémoires sur le Consulat et l'Empire. Droz, GenèveParis, 1971

\section{Summary}

Memoirs of the French Revolution and the Napoleonic Empire sometimes relate to illness and injuries. Being interested in the destiny of such patients we cannot relay exclusively on their own testimony; we need at least one second report. The best documentation would be an account of both-sides the doctor's and the patient's point of view.

Some cases of battle injuries in this period are discussed and compared with the reminiscences of different persons involved. The messages are not always identical-just as nowadays.

$D^{r}$ B. Rüttimann, privat-docent

Clinique orthopédique universitaire du Balgrist, Forchstraße 340, 8008 Zürich, et Institut universitaire d'Histoire de la Médecine, Rämistrasse 71,

8006 Zürich 